\title{
Desarrollo de un entorno virtual colaborativo aplicado a la enseñanza del diseño web, en la ESPOCH Extensión Morona Santiago para mejorar el aprendizaje significativo
}

\section{Development of a collaborative virtual environment applied to web design education, in the ESPOCH Extension Morona Santiago to improve meaningful learning}

Ángel Patricio Flores Orozco. ${ }^{1}$, Paul Xavier Paguay Soxo. ${ }^{2}$, Jonny Israel Guaiña Yungan. ${ }^{3}$ \& Diana Elizabeth Gómez García. ${ }^{4}$
Abstract.
The lack of an educational tool to make web design best practices has resulted in poor academic performance of students in Systems Engineering ESPOCH Extension Morona Santiago why this research is to develop and implement a Virtual Learning Environment Collaborative with characteristics of a HTML editor that allows improve the significant learning and optimize hardware resources available in the institution, the development of EVAC-DW took him out using agile methodology Microsoft Solution Framework, the implemented software was applied to a group of ten students for issuing a kind of theme: creating interactive menus html, in contrast of as many students as a control group limited to the use of traditional tools, then proceeded to the evaluation of knowledge acquired

\footnotetext{
${ }^{1}$ Escuela Superior Politécnica de Chimborazo, Sede Morona Santiago. Macas, Ecuador. aflores@espoch.edu.ec

2 Escuela Superior Politécnica de Chimborazo, Facultad de Informática y Electrónica. Riobamba, Ecuador. ppaguay@espoch.edu.ec

${ }^{3}$ Escuela Superior Politécnica de Chimborazo, Facultad de Informática y Electrónica. Riobamba, Ecuador. jonny.guaina@espoch.edu.ec

${ }^{4}$ Amasoftnia. Desarrollo. Macas, Ecuador. danalizgg713@gmail.com
} 
through a test, practical work and teamwork tools that allow the measurement of significant learning, the results yield an increase in learning by $15 \%$ since the average score that the group reached experimental was 8.63 / 10 versus 7.12 / 10 that obtained the control group, after consumer testing of hardware resource EVAC-DW versus traditional tools we conclude that $10 \%$ less resources needed to use the hardware environment developed, with this is positively proven the hypothesis and the use of EVAC-DW for learning web design is recommended, it should be emphasized that the best feature of the tool is implemented collaborative work in real time.

Keywords: Virtual learning environment, meaningful learning, hardware resource consumption, web application development, html code editor.

\section{Resumen.}

La falta de una herramienta didáctica que permita realizar prácticas adecuadas de diseño web ha provocado bajo rendimiento en los estudiantes de la Carrera de Ingeniería en Sistemas de la ESPOCH Extensión Morona Santiago razón por la cual la presente investigación tiene por objetivo desarrollar y aplicar un Entorno Virtual de Aprendizaje Colaborativo con características de un editor de código html que permita mejorar el aprendizaje significativo y optimizar el recurso hardware. El desarrollo de EVAC-DW se lo llevó a cabo utilizando la metodología ágil Microsoft Solution Framework con un enfoque educativo, el software implementado fue aplicado a un grupo de diez estudiantes durante el dictado de una clase de tema: creación de menus interactivos html, se tuvo en contraparte un grupo de igual número de estudiantes como grupo de control los cuales se limitaron a la utilización de herramientas tradicionales, después se procedió a la evaluación de los conocimientos adquiridos mediante un test, un trabajo práctico y un trabajo en equipo, instrumentos que permitieron la medición del aprendizaje significativo, los resultados obtenidos arrojan el incremento en el aprendizaje significativo en un $15 \%$ ya que el puntaje promedio que alcanzó el grupo experimental fue de 8,63/10 frente a 7,12/10 que obtuvo el grupo de control, los resultados de las pruebas de consumo de hardware de EVAC-DW vs las herramientas tradicionales arrojan que se necesita un 14,5\% menos recursos hardware para la utilización del Entorno desarrollado, con esto queda comprobada de forma positiva la hipótesis planteada y se recomienda el uso de EVAC-DW para el aprendizaje de diseño web, cabe recalcar que la característica que destaca de la herramienta implementada es el trabajo colaborativo en tiempo real. 
Palabras claves: entorno virtual de aprendizaje, aprendizaje significativo, consumo de recursos hardware, desarrollo de aplicaciones web, editor de código html.

\section{Introducción.}

La presente investigación se basa en el desarrollo y aplicación de un entorno virtual de aprendizaje colaborativo, con la finalidad de mejorar el aprendizaje significativo de la asignatura de Aplicaciones Web para la carrera de Ingeniería en Sistemas de la ESPOCH Ext. Morona Santiago, estudio que facilitará la futura aplicación de las TICs en las actividades de enseñanza-aprendizaje en el aula.

El principal objeto de estudio está enfocado en el desarrollo de un entorno virtual colaborativo que de soporte al aprendizaje mediante prácticas de diseño web (html, css, js) individuales y grupales haciendo uso de trabajo colaborativo en tiempo real y de opciones de interacción y comunicación.

El modelo educativo institucional define al ambiente de aprendizaje como: "Escenarios dinámicos para la generación de aprendizajes curriculares, que por sus características de relevancia, pertinencia y significación, deben estar contextualizados, conectados, abiertos y producidos con creatividad, en entornos colaborativos e interculturales." [Modelo Educativo ESPOCH 2014]

Para que se dé el lanzamiento de la herramienta de forma temprana, con resultados tangibles y respuesta ágil y flexible, se ha seguido la metodología Microsoft Solution Framework la cual permite la construcción del producto mientras se modifican y aparecen nuevos requisitos. Quizás no se presente el "producto final”, sino productos que permitan su evolución y mejora.

El rol del maestro, como una parte fundamental del aprendizaje, no es solamente de trasmitir una teoría y conseguir que esta sea memorizada por sus estudiantes sino se busca que guíe, oriente e incentive en la interacción con la nueva información receptada para poder comprenderla y aplicarla en situaciones reales como profesionales, de una manera productiva. Para esto el docente deberá recurrir a la creatividad para impulsar situaciones de aprendizaje en donde el estudiante pueda resolver problemas dentro de un ambiente propicio. De esta interacción entre el docente y estudiante sin duda se enriquecerán los 
dos.

Para ello, es necesario desplegar un ambiente conveniente, donde la conferencia del docente sea una herramienta esencial; así, se establece la situación apropiada para que la interacción con el alumno le incentive a reflexionar, cuestionar e indagar a través de preguntas y el docente las evalúa, cotejando el nivel de comprensión de sus estudiantes, a la vez el alumno aprende a preguntar a otros y preguntarse así mismo, promoviendo el pensamiento crítico.

Los entornos virtuales enfrentan al alumno a un volumen inconmensurable de información. La web 2.0 permite no solamente explorar la información sino modificarla y crear nuevos contenidos. En otras palabras con la web 2.0 el alumno se convierte en alumno-autor. Se basa en una relación de acción-práctica, donde el docente es un facilitador (guía) del aprendizaje así como también diseñador de entornos que motiven y ayuden a alcanzar resultados positivos en el aprendizaje. Estos ambientes otorgan la posibilidad de participar colaborativamente y practicar.

Las características de las herramientas web resultan atractivas para los estudiantes y docentes. Wikis, Blogs y Ambientes virtuales como EVAC-DW (desarrollado en la presente investigación) se utiliza ahora para el aprendizaje. Tradicionalmente un estudiante adquiere su aprendizaje mediante el estudio de libros y la participación en el salón de clases, pero con las herramientas web se sobrepasan estas limitaciones de espacio y tiempo de aprendizaje.

El uso de estas herramientas que aunque específicas, son complementarias para apoyar la creación de comunidades de aprendizaje a la medida de las necesidades de los estudiantes y docentes en el campo del desarrollo web, tales como: EVAC-DW. Esta herramienta creada permite la formación continua, y el aprendizaje es informal ya que se lo puede acceder desde cualquier lugar basta con tener un dispositivo con internet. El tutor-docente tiende a ser un moderador experto en la herramienta y el alumno tiende a ser un creador de contenido.

Los entornos Virtuales de Aprendizaje deben cumplir con las siguientes características: la Dimensionalidad va de acuerdo al espacio de presentación donde lo categorizaremos 
como textual, el Número de usuarios que va en función del número de accesos que pueden utilizar simultáneamente el ambiente virtual, se ha dado en Entorno Virtuales Distribuidos, el Grado de Inmersión se trata del grado y sensación de inmersión que ofrece a sus usuarios. Los sistemas no inmersivos soportan la sensación de "mirar al" entorno virtual, hacen uso de aparatos de interacción convencional como cualquier dispositivo con internet, el Grado de Interactividad permite al usuario explorar y experimentar con el ambiente, modificándolo a este entorno se lo denomina Interactivo.

\section{Metodología desarrollo de EVAC-DW aplicado a la enseñanza del diseño web.}

El sistema que se desarrolla en la presente investigación se lo denominó EVAC-DW (Entorno Virtual de Aprendizaje Colaborativo de Diseño Web) el mismo que sirve de soporte para llevar a cabo la práctica docente en la asignatura de Aplicaciones Web del quinto semestre de la carrera de Ingeniería en Sistemas de la ESPOCH Extensión Morona Santiago.

EVAC-DW cuenta con un entorno de codificación y edición de html, css y javascript además de vista previa en tiempo real, salas colaborativas para trabajo en equipo, material didáctico, ejemplos, ejercicios propuestos y opción para descargar en formato HTML5. Los editores de código cuentan con coloreado para detección de errores e implementan el plugin de codificación abreviada EMMET.

El ingreso a la aplicación se lo hace a través de validación al Sistema Académico Oasis mediante el consumo de Web Services, el rol de autenticación (docente o estudiante) será proporcionado por el Oasis.

\section{Razones que motivan la realización del proyecto}

- Mejorar la calidad de aprendizaje en el tema de diseño web.

- Brindar innovación en herramientas colaborativas que tengan impacto en la enseñanza.

- Utilizar un medio de comunicación global como es el internet para hacer uso de herramientas web que aporten interactividad en el aprendizaje.

\section{Perspectiva del producto}

El sistema puede ser utilizado en la ESPOCH Extensión Morona Santiago como medio 
de enseñanza y aprendizaje ya que cumplirá con todos los objetivos que brinden efectividad y así poder cumplir y obtener los resultados propuestos que demuestren facilidad de adquisición de conocimiento por los estudiantes.

- Se posee disponibilidad en todo momento ya que el acceso a la aplicación se lo hará por medio de la web.

- Proporcionará ayuda en la enseñanza ya que es una herramienta colaborativa en la cual interactúan docentes y estudiantes en un entorno virtual.

\section{Funciones del producto}

Las funciones principales del sistema son:

- Proporcionar trabajo colaborativo por medio de la utilización de salas de trabajo en el entorno virtual.

- Permitir comunicación e interacción en tiempo real de los participantes.

- Aprendizaje de diseño web en el entorno virtual con características de un editor de código básico.

Tabla 1. Herramientas Software Para el Desarrollo

\begin{tabular}{ll}
\hline \multicolumn{1}{c}{ HERRAMIENTA } & \multicolumn{1}{c}{ CARACTERÍSTICA } \\
\hline WINDOWS 8 & $\begin{array}{l}\text { Sistema Operativo } \\
\text { Metodología de Desarrollo de Soft- } \\
\text { ware }\end{array}$ \\
Microsoft Solution Framework (MSF) & $\begin{array}{l}\text { Lenguaje de Modelado } \\
\text { Unified Modeling Language (UML) } \\
\text { Python }\end{array}$ \\
vices
\end{tabular}

\section{Requerimientos funcionales de EVAC-DW}

A continuación se detalla cada uno de los requerimientos funcionales dispuestos a cumplir con la aplicación EVAC-DW en los cuales se toman en cuenta las entradas, procesos y salidas. 
1. Gestionar ejemplos: EVAC-DW debe otorgar y permitir crear, modificar o eliminar ejemplos por parte del docente y dará acceso limitado al estudiante para que solo pueda crear ejemplos en la aplicación.

2. Gestionar material: EVAC-DW podrá dar el permiso necesario para que el docente pueda gestionar su material didáctico como crear, modificar o eliminar y limitará al estudiante para que solo pueda crear material.

3. Trabajo colaborativo: El sistema EVAC-DW debe proporcionar el mecanismo necesario para trabajar colaborativamente debe permitir escoger salas de trabajo con el fin de interactuar significativamente entre los participantes para tener una vista previa de la página y comunicarse entre sí.

4. Usar editores: El sistema de proporcionar el uso de editores orientados al diseño web como HTML, CSS o JAVASCRIPT ya que debe permitir codificar en estos editores para visualizar la vista previa del diseño en tiempo real.

5. Descargar la página web creada con EVAC-DW: El sistema permite codificar en editores orientados a diseño web y por ende debe proporcionar y permitir la opción para descargar el archivo desarrollado a su computador.

\section{Diseño de la Investigación}

Para medir los requerimientos hardware de EVAC-DW se utilizó el administrador de tareas de Windows, estos datos serán comparados con los requerimientos mínimos que exigen las herramientas tradicionales de diseño web.

Para medir el aprendizaje significativo al cual se llega con el uso de la herramienta se lo hará de la siguiente manera:

- Contar con dos grupos de estudiantes al azar, de igual número de participantes, pertenecientes a la Carrera de Ing. en Sistemas de la ESPOCH-MS.

- A los dos grupos se le impartió al mismo tiempo un tema de clase concerniente a diseño web.

- El grupo de intervención experimental utilizó EVAC-DW mientras el grupo de control lo hizo con las herramientas tradicionales de diseño web.

- Al final de la clase se evaluaron tres parámetros: test de conocimientos, taller individual y un trabajo en equipo.

- Los datos recogidos de dichas evaluaciones se someterán a un análisis estadístico para la verificación de la hipótesis $\mathrm{H} 1$.

\section{HIPÓTESIS}

H1.- El uso de un entorno virtual de aprendizaje colaborativo mejora el requerimiento 
hardware y el aprendizaje significativo de los estudiantes de la carrera de Ingeniería en

Sistemas de la ESPOCH Extensión Morona Santiago en el ámbito del Diseño Web.

Tabla 2.Operacionalización de variables

\begin{tabular}{|c|c|c|c|c|}
\hline VARIABLE & $\begin{array}{l}\text { CATEGO- } \\
\text { RÍA }\end{array}$ & INDICADOR & TÉCNICA & $\begin{array}{c}\text { FUENTE DE VERIFICACIÓN } \\
\text { /INSTRUMENTO }\end{array}$ \\
\hline \multirow[t]{3}{*}{$\begin{array}{l}\text { Requerimien- } \\
\text { tos Hardware }\end{array}$} & \multirow[t]{3}{*}{ Dependiente } & $\begin{array}{l}\text { INDICADOR } \\
1: \text { RAM }\end{array}$ & \multirow[t]{3}{*}{$\begin{array}{l}\text { - Observación Directa } \\
\text { - Monitoreo } \\
\text { - Recopilación de in- } \\
\text { formación. }\end{array}$} & \multirow[t]{3}{*}{$\begin{array}{l}\text { - Administrador de Tareas. } \\
\text { - Monitor de sucesos } \\
\text { - Especificaciones Técnicas } \\
\text { - Estadística descriptiva, }\end{array}$} \\
\hline & & $\begin{array}{l}\text { INDICADOR } \\
\text { 2: Disco Duro }\end{array}$ & & \\
\hline & & $\begin{array}{l}\text { INDICADOR } \\
\text { 3: CPU }\end{array}$ & & \\
\hline \multirow{3}{*}{$\begin{array}{l}\text { Aprendizaje } \\
\text { Significativo }\end{array}$} & \multirow{3}{*}{ Dependiente } & $\begin{array}{l}\text { INDICADOR } \\
\text { 4: Puntaje obte- } \\
\text { nido en Saber }\end{array}$ & \multirow[t]{3}{*}{$\begin{array}{l}\text { - Test (saber) } \\
\text { - Taller Individual } \\
\text { (saber hacer) } \\
\text { - Trabajo en equipo } \\
\text { (saber ser) }\end{array}$} & \multirow[t]{3}{*}{$\begin{array}{l}\text { - Calificación del Test } \\
\text { - Calificación del taller in- } \\
\text { dividual } \\
\text { - Calificación del trabajo } \\
\text { en equipo } \\
\text { - Prueba t student }\end{array}$} \\
\hline & & $\begin{array}{l}\text { INDICADOR } \\
\text { 5: Puntaje obte- } \\
\text { nido en Saber } \\
\text { hacer }\end{array}$ & & \\
\hline & & $\begin{array}{l}\text { INDICADOR } \\
\text { 6: Puntaje obte- } \\
\text { nido en Saber } \\
\text { ser }\end{array}$ & & \\
\hline \multirow[b]{2}{*}{ EVAC-DW } & \multirow{2}{*}{$\begin{array}{l}\text { Indepen- } \\
\text { diente }\end{array}$} & $\begin{array}{l}\text { Cantidad de es- } \\
\text { tudiantes }\end{array}$ & \multirow[t]{2}{*}{$\begin{array}{l}\text { - Observación Di- } \\
\text { recta }\end{array}$} & \multirow[t]{2}{*}{ - Registro de asistencia } \\
\hline & & $\begin{array}{l}\text { Porcentaje de } \\
\text { Aceptación de } \\
\text { la Herramienta }\end{array}$ & & \\
\hline
\end{tabular}

Fuente: Autores

Elaborado por: Los autores

\section{Población y Muestra}

Al tratarse de una hipótesis que cuenta con dos variable dependientes distintas será necesario tener dos grupos de estudio completamente diferentes.

- La primera población (variable dependiente 1) serán los datos que se pueda recabar del consumo de recursos hardware mediante el Administrador de Tareas y el Monitor de Recursos tanto de EVAC-DW como de los editores elegidos, 
como se trata de una gran cantidad de datos que puedan ser medidos utilizaremos un método no probabilístico. Los datos fueron tomados cada minuto durante una hora, es así que se obtuvieron 60 datos para cada indicador (memoria, disco y $\mathrm{cpu})$.

- La segunda población (variable dependiente 2) para el presente experimento son los estudiantes de la carrera de Ingeniería en Sistemas de la ESPOCH Extensión Morona Santiago en los cuales se midió el aprendizaje significativo alcanzado con el uso de EVAC-DW, cantidad que asciende a 21 estudiantes distribuidos en sexto, octavo y décimo semestre. Debido a la poca cantidad de población no se hizo un cálculo para obtener la muestra y se trabajó con toda la población.

- Al momento de la realización del experimento se contó con la presencia de 20 estudiantes.

\section{Procedimientos Generales}

Con el objetivo de hallar las características y requerimientos hardware de editores de código y entornos de desarrollo se procedió a elegir ciertas aplicaciones que se enmarcan en el presente estudio es así que mediante consulta en foros y blogs se encontraron las más utilizadas por los desarrolladores y esencialmente que cumplan con las principales características de un editor de código compatible con HTML5.

Para obtener los datos del consumo de recursos hardware se procedió a instalar todos y cada uno de los editores seleccionados. Los mismos que fueron utilizados para la creación de un sitio web básico y al mismo tiempo medir el consumo de recursos mediante observación directa del Administrador de Tareas y el Monitor de Recursos, este proceso se lo hizo durante una hora para poder obtener 60 datos tanto del consumo de Memoria, Disco y CPU, como se seleccionaron 6 editores se han tomado 10 datos por cada editor. Para la aplicación de EVAC-DW se procedió a desplegarlo en un servidor de pruebas proporcionado por Heroku (computación en la nube), luego de la realización de pruebas se detectaron algunos errores y se incrementaron funcionalidades.

Posteriormente para la toma de datos que contribuyen a medir el aprendizaje significativo (saber, saber hacer, saber ser) se procedió de la siguiente manera:

- Planificar un tema de clase.

- Crear un test que mida el parámetro Saber. 
- Proponer un ejercicio para ser resuelto individualmente al final de la clase que luego pueda ser evaluado y mida el parámetro Saber hacer.

- Proponer un trabajo en grupo que permita medir el Saber ser.

- Convocar a todos los estudiantes de la carrera de Ingeniería en Sistemas para el dictado del tema de clase.

- Crear dos grupos al azar tomando en cuenta el nivel cada uno con 10 estudiantes.

- Sortear un grupo para que durante el desarrollo de la clase utilice EVAC-DW mientas que el otro grupo puede utilizar cualquier herramienta de codificación y desarrollo.

- Aplicar las herramientas de medición del aprendizaje significativo (saber, saber hacer, saber ser), al final del dictado de la clase.

\section{RESULTADOS OBTENIDOS DEL CONSUMO DE RECURSOS HARDWARE}

Los datos que se muestran a continuación se obtuvieron por observación directa al Administrador de Tareas y al Monitor de Recursos que proporciona Windows 8.1, los datos fueron tomados cada minuto con la ayuda de un colaborador, mientras se diseñaba una página web básica, utilizando los tres navegadores más populares en Windows: Firefox 42, Chrome v 47.0.2526.80 m, Internet Explorer 11.

Tabla 3. Porcentaje de uso de HW

\begin{tabular}{lccccc}
\hline & N & Máximo & Media & $\begin{array}{l}\text { Porcentaje de } \\
\text { uso }\end{array}$ & $\begin{array}{l}\text { Diferencia } \\
\text { de Porcenta- } \\
\text { jes }\end{array}$ \\
& Estadístico & Estadístico & Estadístico & Estadístico & \\
\hline $\begin{array}{l}\text { EVAC-DW Uso de } \\
\text { Memoria en MB }\end{array}$ & 60 & 540 & 81,1667 & 15,03087037 & $\begin{array}{l}10,86 \% \text { me- } \\
\text { nos memoria }\end{array}$ \\
$\begin{array}{l}\text { EDITORES Uso de } \\
\text { Memoria en MB }\end{array}$ & 60 & 540 & 139,8317 & 25,89475926 & \\
$\begin{array}{l}\text { EVAC-DW Uso de } \\
\text { Disco en MB/s } \\
\text { EDITORES Uso de } \\
\text { Disco en MB/s }\end{array}$ & 60 & 46 & 0,1117 & 0,242826087 & $\begin{array}{l}\mathbf{1 4 , 5} \% \text { menos } \\
\text { recursos HW }\end{array}$ \\
$\begin{array}{l}\text { EVAC-DW Porcen- } \\
\text { taje de Uso de CPU }\end{array}$ & 60 & 46 & 3,8833 & 8,441956522 & $\begin{array}{l}8,22 \% \text { me- } \\
\text { nos disco }\end{array}$ \\
$\begin{array}{l}\text { EDITORES Porcen- } \\
\text { taje de Uso de CPU }\end{array}$ & 60 & 91 & 8,4967 & 9,337032967 & \\
$\begin{array}{l}\text { N válido (por lista) } \\
\text { N }\end{array}$ & 60 & 91 & 30,5933 & 33,61901099 & $\begin{array}{l}\text { nos \% de } \\
\text { cpu }\end{array}$ \\
\hline
\end{tabular}


Figura 1. Consumo de Recursos Hardware

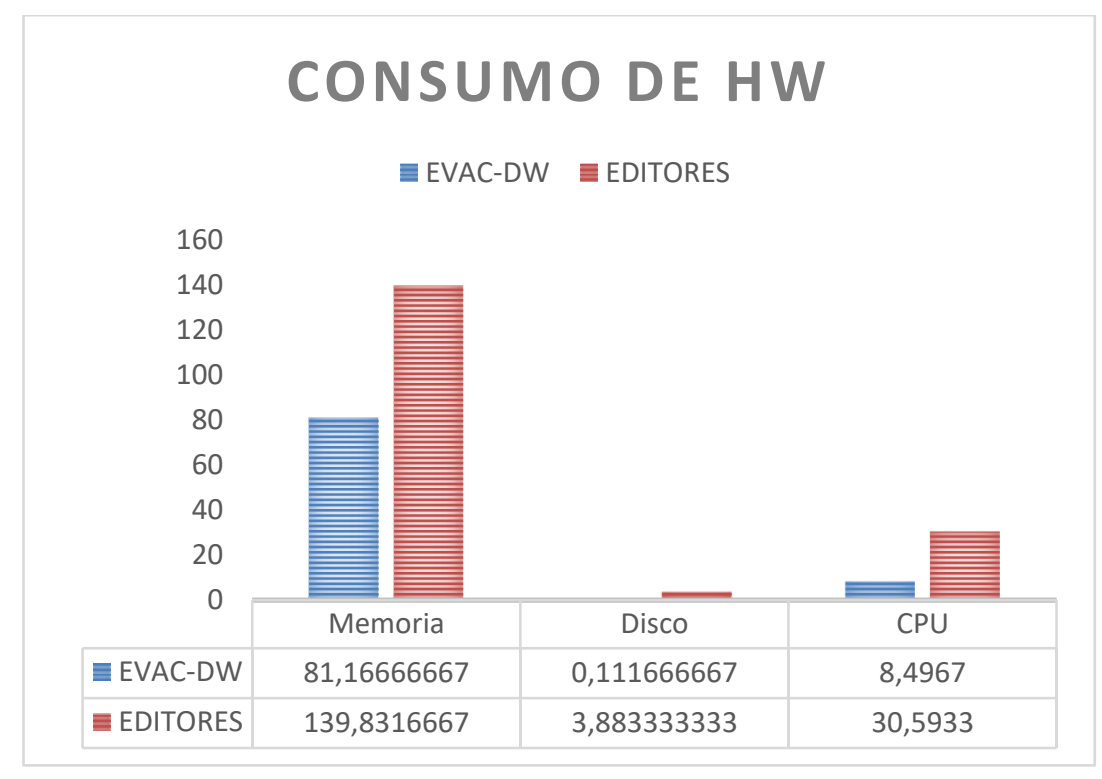

Los resultados del análisis de estadísticos descriptivos claramente dejan ver que el uso de EVAC-DW disminuye la necesidad de recursos hardware frente al uso de herramientas tradicionales de codificación para diseño web, es así que el promedio de memoria que utilizan las herramientas tradicionales es de $140 \mathrm{MB}$ frente a $81 \mathrm{MB}$ que utiliza EVACDW reduciendo la necesidad en $59 \mathrm{MB}$. El promedio de uso de disco que necesitan las herramientas tradicionales es de $3,88 \mathrm{MB} / \mathrm{s}$ frente a $0,11 \mathrm{MB} / \mathrm{s}$ que necesita EVAC-DW reduciendo la necesidad de 3,77 MB/s. El porcentaje de uso de CPU que utilizan las herramientas tradicionales es de 2,95\% frente a 0,16\% que utiliza EVAC-DW reduciendo la necesidad de 2,79 \% de CPU. Hay que tomar en cuenta que la medida de \% de uso de CPU es relativa al procesador del equipo en donde se realizó la toma de los datos.

El bajo consumo de recursos hardware que muestra EVAC-DW permite su uso en casi cualquier dispositivo que tenga conexión a internet mediante un navegador actualizado, razón por la cual la herramienta fue desarrollada con vistas responsivas que se adapten a cualquier tamaño de pantalla.

Existen valores altos en el error estándar, en la media, asimetría y curtosis debido a que los datos tomados para medir el consumo de recursos hardware de los editores tradicionales no son de una solo herramienta sino de seis pero para calcular los promedios 
es necesario hacerlo de todas.

Para hallar porcentajes de efectividad se utiliza el valor máximo de cada sub variable (MEMORIA, DISCO, CPU) siendo estos el 100\% de HW requerido y los valores promedio lo que habitualmente consumen.

Resultados obtenidos para medir el aprendizaje significativo

\begin{tabular}{llrlr}
\hline & & & & \\
& GRUPO 1 CON EVAC-DW & & \\
& ESTU- & TEST & $\begin{array}{l}\text { TA- } \\
\text { LLER }\end{array}$ & $\begin{array}{l}\text { TRAB. COLA- } \\
\text { BORATIVO }\end{array}$ \\
& DIANTE & & 8 & 9 \\
& SRMJ & 8,66 & 8 & 9 \\
GRUPO 1.1 PARA & GREJ & 7,33 & 10 & 9 \\
TRABAJO COLABO- & TCBG & 7,5 & 9 & 9 \\
RATIVO & ALKM & 7,16 & 9 & 9 \\
& UBFG & 9,66 & 10 & 8 \\
GRUPO 1.2 PARA & OBRE & 8 & 9 & 8 \\
TRABAJO COLABO- & CLPA & 7,16 & 10 & 8 \\
RATIVO & NRTA & 7,33 & 9 & 8 \\
& HJJC & 10 & 10 & 8,5 \\
PROMEDIO & & 8,08 & 9,3 & 8 \\
\hline
\end{tabular}

GRUPO 2 CON HERRAMIENTAS TRADICIONALES

\begin{tabular}{|c|c|c|c|c|}
\hline & & TEST & $\begin{array}{l}\text { TA- } \\
\text { LLER }\end{array}$ & $\begin{array}{l}\text { TRAB. COLA- } \\
\text { BORATIVO }\end{array}$ \\
\hline \multirow{6}{*}{$\begin{array}{l}\text { GRUPO } 2.1 \text { PARA } \\
\text { TRABAJO COLABO- } \\
\text { RATIVO }\end{array}$} & MAEE & 7,83 & 7 & \\
\hline & PMCJ & 8 & 9 & \\
\hline & GBWT & 8,33 & 8 & \\
\hline & PLSI & 7,66 & 9 & \\
\hline & PJCF & 6,83 & 7 & \\
\hline & ZBBA & 8,33 & 7 & \\
\hline \multirow{4}{*}{$\begin{array}{l}\text { GRUPO } 2.2 \text { PARA } \\
\text { TRABAJO COLABO- } \\
\text { RATIVO }\end{array}$} & APWR & 9 & 8 & \\
\hline & RVDL & 8,82 & 7 & \\
\hline & STAV & 7,66 & 2 & \\
\hline & CSMG & 7 & 0 & \\
\hline PROMEDIO & & 7,95 & 6,4 & \\
\hline
\end{tabular}

Fuente: Trabajo experimental

Elaborado por: Los autores 
Figura. 2 Promedio de Calificaciones para medir el Aprendizaje Significativo

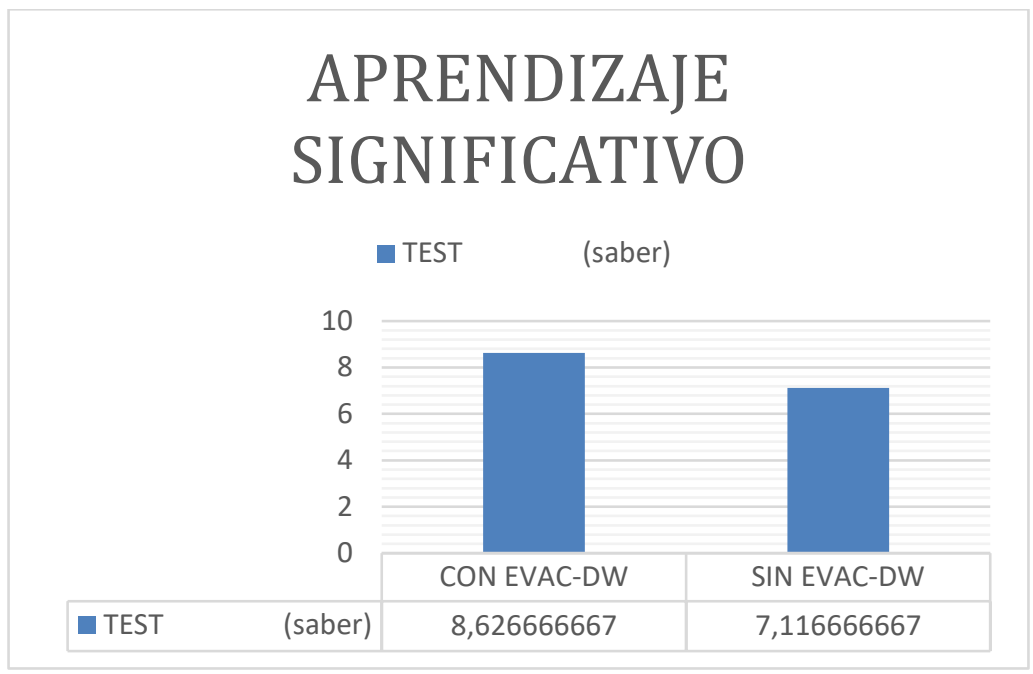

Si hallamos un promedio general de aprendizaje significativo de los dos grupos podemos observar que el grupo experimental que utilizó EVAC-DW alcanzó una calificación mayor que el grupo de control, el aprendizaje significativo se incrementó en un $15 \%$, los promedio obtenidos fueron de $8,63 / 10$ para el grupo experimental y 7,12/10 para el grupo de control.

\section{Discusión y comprobación de la hipótesis}

La prueba t-student será usada para comprobar las dos variables por separado Como el valor de significancia de las dos variables es menor que $\boldsymbol{\alpha}=\mathbf{0 , 0 5}$ para las dos variables dependientes queda aceptada la hipótesis alternativa: H1:"El uso de un entorno virtual de aprendizaje colaborativo mejora el requerimiento hardware y el aprendizaje significativo de los estudiantes de la carrera de Ingeniería en Sistemas de la ESPOCH Extensión Morona Santiago en el ámbito del Diseño Web.” y rechazada la nula: "El uso de un entorno virtual de aprendizaje colaborativo no mejora el requerimiento hardware ni el aprendizaje significativo de los estudiantes de la carrera de Ingeniería en Sistemas de la ESPOCH Extensión Morona Santiago en el ámbito del Diseño Web.”

\section{Conclusiones}

Del análisis de los datos obtenidos durante el uso y aplicación de EVAC-DW tanto para consumo de recursos como para medir el aprendizaje significativo se pudo establecer las siguientes conclusiones: 
- Después de analizar las herramientas tradicionales de desarrollo nos encontramos de que estas herramientas fueron creadas con fines de producción mas no educativos por lo cual es necesario que exista una herramienta que sirva para la adquisición de conocimiento de diseño Web como es el caso de EVAC-DW motivo de este presente estudio.

- Cuando el estudiante empieza en la adquisición del conocimiento de diseño web es necesario que cuente con una herramienta que le permita practicar las etiquetas html, ya que de esta forma el estudiante construye el conocimiento se interioriza y llega a ser significativo.

- Los lenguajes programación libres y el software libre en general permiten llegar a la implementación de grandes aplicaciones web ya que a lo largo del tiempo se ha popularizado y ha ganado grande adeptos que han logrado la consolidación de dichas herramientas o lenguajes, es el caso de JAVASCRIPT que en la actualidad es un excelente lenguaje para la creación del backend en aplicaciones web por lo cual se lo utilizó para la creación de EVAC-DW, con la ayuda de NODEJS que es un framework basado en javascript.

- Después de tomar datos durante el uso de EVAC-DW y analizarlos podemos concluir que la herramienta motivo de la presente investigación consume un 14,5\% menos recursos hardware en comparación con las herramientas tradicionales de desarrollo.

- El uso de EVAC-DW permite que los estudiantes mejoren su aprendizaje significativo en un $15 \%$ ya que el puntaje promedio que alcanzó el grupo experimental fue de 8,63/10 frente a 7,12/10 que obtuvo el grupo de control.

- El uso de una herramienta colaborativa permite que los estudiantes cumplan con los trabajos en equipo a cabalidad, ya que evita que tengan que desplazarse a un lugar de reunión y tengan que estar presentes físicamente, esta funcionalidad de EVAC-DW ha hecho que despunte sobre las herramientas tradicionales de codificación web.

\section{Referencias Bibliográficas}

- CHERRE, Rafael. (2002) Manual del Programador Web, $3^{\text {a }}$ ed. Lima-Perú, Editorial Macro, pp. 204-278.

- COOL, César; MARTÍN, Elena; MAURI, Teresa; MIRAS Mariana. (2007) El constructivismo en el aula. $18^{\mathrm{a}}$ ed. Barcelona-España, Editorial GRAO, pp. 14-19. 
- DIAZ, M.; PEREZ, M.; GRIMMÁN A,; MENDOZA L. (2006) Propuesta de una metodología de desarrollo de software educativo bajo un enfoque de calidad sistémica. [en línea] Caracas- Venezuela. Universidad Simón Bolívar. [Consulta: 22 septiembre 2015]. Disponible en: http://www.academia-interactiva.com/doc/ise.pdf

- ESPOCH DDA. (2014) Modelo Educativo ESPOCH 2014, RiobambaEcuador, pp. 1-48.

- FLORES, Angel (2015), Desarrollo de un entorno virtual colaborativo aplicado a la enseñanza del diseño web, en la ESPOCH extensión Morona Santiago para mejorar el aprendizaje significativo, Riobamba - Ecuador., Instituto de Posgrado y Educación Continua, Escuela Superior Politécnica de Chimborazo., Tesis., 108p.

- GAUCHAT, Juan. (2012) EL GRAN LIBRO DE HTML5, CSS3 Y JAVASCRIPT, Primera Edición, Barcelona-España, Editorial MARCOMBO, pp. 25-70.

- GONZALES, O.; FLORES, M. (2000) El trabajo docente: enfoques innovadores para el diseño de un curso. $3^{\mathrm{a}}$ ed. México DF- México, Editorial TRILLAS, pp. 12-34.

- LÓPEZ, José. (2003) Domine HTML y DHTML, $1^{\mathrm{a}}$ ed, México-México, Alfaomega, pp. 35-95.

- MORAS, A. (2011) "Proceso de enseñanza-aprendizaje y web 2.0: valoración del conectivismo como teoría de aprendizaje postconstructivista". Las Tecnologías de la Información y de la Comunicación (TIC) y los nuevos contextos de aprendizaje [en línea], (España) 20, pp. 117-140. [Consulta: 17 septiembre 2015]. ISSN: 1578-7001. Disponible en: http://dadun.unav.edu/handle/10171/18344

- MUSCIANO, C; KENNEDY, B. (1999) HTML la guía completa, $1^{a}$ ed, México-México, Editorial McGraw-Hill, pp. 102-187.

- OROS, Juan. (2010) Diseño de Páginas Web con XHTML, JavaScript y CSS, $2^{\mathrm{a}}$ ed. Madrid- España, Editorial RA-MA, pp. 35-90.

- OROS, Juan. (2000) Diseño de Páginas Web interactivas con Java Script: navega por internet, $1^{\mathrm{a}}$ ed. México-México, Editorial Alfaomega, pp. 205290.

- PILGRIM, Mark. (2010) HTML5 UP AND RUNNING, $1^{\mathrm{a}}$ ed, Estado Unidos de América, Editorial O’REILLY Google Press, pp. 20-108.

- PIMIENTA, Julio. (2008) Constructivismo: Estrategias para aprender a aprender. $3^{\mathrm{a}}$ ed. México-México, Editorial Pearson Educación, pp. 68-200.

- PIMIENTA, Julio. (2007) Metodología Constructivista: Guía para la Planeación Docente. $2^{\mathrm{a}}$ ed. México-México, Editorial Pearson Educación, pp. 100-135.

- SCHANK, Roger. (1997) Un enfoque revolucionario dirigido a formar equipos de trabajo altamente capacitados, $1^{\mathrm{a}}$ ed, México-México, Editorial McGraw-Hill, pp. 115-175. 
Para citar el artículo indexado.

Flores Orozco, Ángel, Paguay Soxo, P., Paguay Soxo, P., \& Guaiña Yungan, J. (2019). Desarrollo de un entorno virtual colaborativo aplicado a la enseñanza del diseño web, en la ESPOCH Extensión Morona Santiago para mejorar el aprendizaje significativo. Explorador Digital, 3(3.1), 77-92. https://doi.org/10.33262/exploradordigital.v3i3.1.866

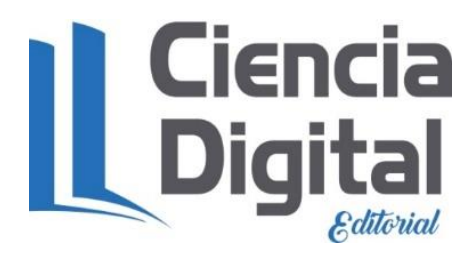

El artículo que se publica es de exclusiva responsabilidad de los autores y no necesariamente reflejan el pensamiento de la Revista Explorador Digital.

El articulo queda en propiedad de la revista y, por tanto, su publicación parcial y/o total en otro medio tiene que ser autorizado por el director o editor de la Revista Explorador Digital.
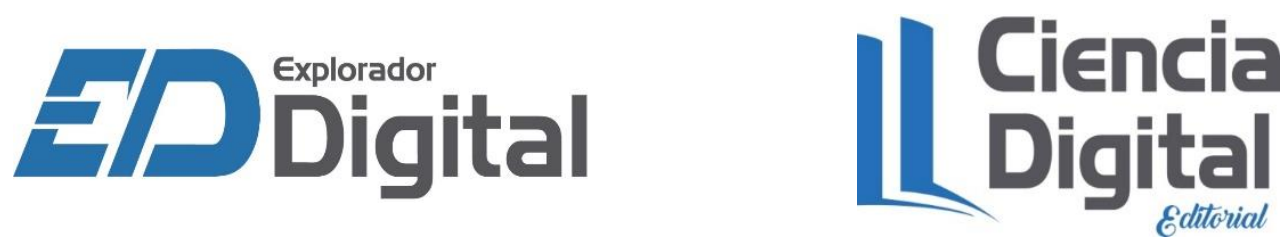